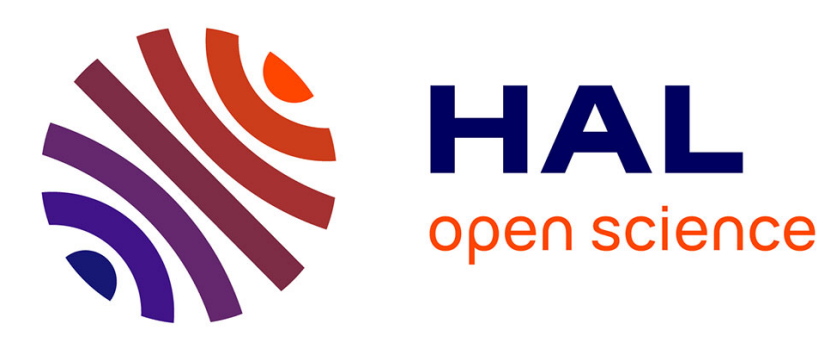

\title{
Ultimate regime in Rayleigh-Bénard convection: The role of plates
}

Francesca Chillà, Marie Rastello, Sébastien Chaumat, Bernard Castaing

\section{To cite this version:}

Francesca Chillà, Marie Rastello, Sébastien Chaumat, Bernard Castaing. Ultimate regime in RayleighBénard convection: The role of plates. Physics of Fluids, 2004, pp.2452-2456. 10.1063/1.1751396 . hal-00620829

\section{HAL Id: hal-00620829 \\ https://hal.science/hal-00620829}

Submitted on 8 Sep 2011

HAL is a multi-disciplinary open access archive for the deposit and dissemination of scientific research documents, whether they are published or not. The documents may come from teaching and research institutions in France or abroad, or from public or private research centers.
L'archive ouverte pluridisciplinaire HAL, est destinée au dépôt et à la diffusion de documents scientifiques de niveau recherche, publiés ou non, émanant des établissements d'enseignement et de recherche français ou étrangers, des laboratoires publics ou privés. 


\title{
Ultimate regime in Rayleigh-Bénard convection: The role of plates
}

\author{
F. Chillà, ${ }^{a)}$ M. Rastello, S. Chaumat, and B. Castaing \\ Ecole Normale Supérieure de Lyon, 69364 Lyon Cedex 7, France
}

(Received 21 October 2003; accepted 5 March 2004; published online 1 June 2004)

\begin{abstract}
The ultimate regime of convection, long ago predicted by Kraichnan [Phys. Fluids 5, 1374 (1962)], could be called elusive because some apparently equivalent experiments showed it while others did not, with no apparent reasons for this discrepancy. In this paper, we propose a model which accounts for the finite heat conductivity and heat capacity of real active boundaries. Bad thermal characteristics of the plates can explain differences between various experiments, in agreement with recent numerical simulations. (C) 2004 American Institute of Physics. [DOI: 10.1063/1.1751396]
\end{abstract}

\section{INTRODUCTION}

The ultimate regime of convection, predicted by Kraichnan ${ }^{1,2}$ more than 40 years ago, recently raised considerable interest. Different groups $^{3-6}$ obtained experimental apparently contradictory results. Seemingly equivalent experiments showed this ultimate regime or not, with no apparent reasons for this discrepancy. This paper proposes an explanation.

In Rayleigh-Bénard convection, the working fluid is limited vertically by two heat conducting plates, the temperature of the top plate $(T-\Delta / 2)$ being smaller than the bottom one $(T+\Delta / 2)$. Nondimensional numbers parametrizing the equations and boundary conditions for the fluid are as follows.

(i) Prandtl number,

$\operatorname{Pr}=\nu / \kappa_{f}$,

where $\nu$ is the kinematic viscosity and $\kappa_{f}$ is the heat diffusivity.

(ii) Rayleigh number,

$$
\mathrm{Ra}=g \alpha \Delta h^{3} / \nu \kappa_{f},
$$

where $\alpha$ is the isobaric coefficient of thermal expansion, $g$ is the gravitational acceleration, and $h$ is the height of the cell.

(iii) Aspect ratio,

$$
\Gamma=d / h,
$$

where $d$ is, for instance, the diameter of a cylindrical cell.

The Nusselt number is the ratio between the heat flux $Q$ and what it would be for a quiescent fluid,

$$
\mathrm{Nu}=Q h /\left(\lambda_{f} \Delta\right),
$$

where $\lambda_{f}$ is the fluid's thermal conductivity. Due to dimensional similarity, $\mathrm{Nu}$ should depend only on nondimensional numbers.

To make a long story short, let us say that most experimental results are in fair agreement with a law,

${ }^{a)}$ Electronic mail: francesca.chilla@ens-lyon.fr

$$
\mathrm{Nu} \propto \mathrm{Ra}^{1 / 3},
$$

corresponding to the temperature gradients being concentrated close to the plates, and the flow introducing a relatively poor coupling between these plates. As can be seen from the definitions, the height $h$ then disappears from the relation between the flux $Q$ and the temperature difference $\Delta$. According to a recent extensive theoretical study, ${ }^{7}$ the approximative agreement with Eq. (1) is due to a series of crossovers between various regimes. On the contrary, in the ultimate turbulent regime, the dissipative coefficients $\nu$ and $\kappa_{f}$ should appear in the $Q$ versus $\Delta$ relation only through their ratio $\mathrm{Pr}$, giving the following asymptotic law:

$$
\mathrm{Nu} \propto \mathrm{Ra}^{1 / 2} f(\mathrm{Pr}) .
$$

For $\operatorname{Pr}>1$, Kraichnan ${ }^{1}$ proposed the relation,

$$
\mathrm{Nu} \propto \frac{\mathrm{Ra}^{1 / 2} \operatorname{Pr}^{-1 / 4}}{\ln \mathrm{Ra}^{3 / 2}},
$$

where the logarithmic correction comes from the logarithmic profile of turbulent boundary layers and the dependence of the size of the viscous sublayer on the Reynolds number Re and thus on $\mathrm{Ra}^{8}{ }^{8}$ Such logarithmic corrections occur also for turbulent drag on a body.

Chavanne et al. first reported experimental observations, ${ }^{4,9}$ showing an increase in the logarithmic slope $\partial \ln \mathrm{Nu} / \partial \ln \mathrm{Ra}$ for $\mathrm{Ra}>10^{11}$. Later, Roche et al. ${ }^{10}$ showed that rough boundaries give the pure asymptotic law [Eq. (2)] on more than one decade. This agrees with Eq. (3) if the rough boundaries suppress the logarithmic corrections, as in the drag case. However, Wu et al. ${ }^{3}$ previously explored the same range of $\mathrm{Ra}$, finding no change in the $\mathrm{Nu}$ versus $\mathrm{Ra}$ relation of Eq. (1). Niemela et al. ${ }^{5}$ similarly found a single power law: $\mathrm{Nu}=0.128 \mathrm{Ra}^{0.309}$, up to $\mathrm{Ra}=10^{17}$.

All these experiments used cells of aspect ratio 1/2. Recently, Niemela et al. ${ }^{6}$ also observed an increase in the logarithmic slope $\partial \ln \mathrm{Nu} / \partial \ln \mathrm{Ra}$ in a cell of aspect ratio 1 . Other authors reported departure from the law given by Eq. (1), but on very limited Ra range. ${ }^{11,12}$

The important question is now to understand the apparent discrepancy between these observations. In a recent paper, we examined the influence of both finite heat capacity 
and finite thermal conductivity for the plates ${ }^{13}$ (Hunt et al. also considered this question), ${ }^{14}$ but could not establish a clear difference between the results of various groups. Recent remarks of Niemela et ll. $^{6}$ on Chavanne's data and numerical simulations of Verzicco, ${ }^{15}$ showing the influence of plate thermal conductivity on the flow, led us to reconsider the question.

This paper is organized as follows. We first give an argument showing that the plates properties can modify the $\mathrm{Nu}$ versus $\mathrm{Ra}$ relation. We then present a model to quantify this argument. We improve our previous result, avoiding an earlier approximation. Finally, we compare to experiment, accounting for the remark of Niemela et al.

\section{THE ARGUMENT}

Imagine a plume starting from the hot (bottom) plate. It carries heat, and thus tends to cool the plate, at its source. The lower surface temperature of the plate, the lower the average temperature of the fluid carried by the plume. If the plume temperature is lower than the temperature at any point in the surrounding fluid, the plume cannot rise further. Let us call $\delta_{c}$ the amplitude of the surface temperature variation which stops the development of a plume. A precise evaluation of $\delta_{c}$ will be proposed later (Sec. IV), based on this definition.

Now, consider a plate whose heat capacity and thermal conductivity are so low that they cannot maintain a constant uniform temperature. The heat capacity of the fluid in the bottom boundary layer will be all that maintains the heat flux of the plume. This heat flux will thus persist for the boundary layer's characteristic diffusion time, i.e., $\tau=\xi^{2} / \kappa_{f}$ where $\xi$ $=h / 2 \mathrm{Nu}$ is the boundary layer thickness.

For low plate thermal conductivity and heat capacity, the plume can rise freely only during the period $\tau$. During this time, it rises to an approximate maximum height,

$$
\zeta=g \alpha \Delta \tau^{2}=\left(g \alpha \Delta / \kappa_{f}^{2}\right) \xi^{4}=\xi\left(\operatorname{Ra} \operatorname{Pr} / 8 \mathrm{Nu}^{3}\right) .
$$

Obviously, the height of the plume is larger than the size of the thermal boundary layer $(\zeta>\xi)$, and thus

$$
\mathrm{Nu}^{3}<\operatorname{Ra} \operatorname{Pr} / 8 .
$$

Thus, if the plate is unable to maintain its temperature, $\mathrm{Nu}$ cannot rise more rapidly than $\mathrm{Ra}^{1 / 3}$ and the ultimate regime cannot develop. In the following section, we try to quantify this argument, by determining the plate's surface temperature distribution for finite heat capacity and thermal conductivity.

\section{THE MODEL}

To estimate this distribution, we consider a twodimensional model for the bottom plate. Symmetric arguments apply to the cold top plate. We model the plate as a horizontal, $x$-infinite plate, bounded by the planes $z=0$ and $z=-a$. At the top of the plate, we superimpose a periodic heat flux on the uniform, average vertical flux. The periodic heat flux represents the effect of plumes. The average, verti- cal flux corresponds to the experiment's uniform constant heat flux across the bottom of the plate. Thus the temperature distribution in the plate, $\theta(x, z, t)$, is

$$
\theta(x, z, t)=T_{S}-{ }_{z} G_{o}+\tilde{T}(x, z, t),
$$

where $T_{S}$ is the average temperature at the bottom of the fluid. $-G_{o}$ is the average gradient resulting from the average applied heat flux, $\tilde{T}(x, z, t)$ is the difference between the temperature in the plate and its $z$-dependent average and

$$
\left(\partial_{z} \tilde{T}\right)_{z=0}=G_{p} e^{i(k x-\omega t)},
$$

with $-\lambda_{p} \partial_{z} \tilde{T}$ ( $\lambda_{p}$ is the plate thermal conductivity) being the superimposed flux that mimics the plumes. $\widetilde{T}$ obeys the Fourier heat equation,

$$
\partial_{t} \tilde{T}=\kappa_{p} \Delta \widetilde{T},
$$

where $\kappa_{p}$ is the heat diffusivity in the plate. At the bottom of the plate, the vertical heat flux is $-G_{o}$, so, $\left(\partial_{z} \widetilde{T}\right)_{z=-a}=0$. Looking for a solution of the form $\widetilde{T}=T(z) e^{i(k x-\omega t)}$, one obtains

$$
d^{2} T / d z^{2}=q^{2} T,
$$

with $q^{2}=k^{2}-i\left(\omega / \kappa_{p}\right)$. Solving with the above boundary conditions gives

$$
T(z=0)=\left(G_{p} / q\right) \frac{e^{q a}+e^{-q a}}{e^{q a}-e^{-q a}} .
$$

Let us call $H(q a)=\left|q a\left(e^{q a}-e^{-q a}\right) /\left(e^{q a}+e^{-q a}\right)\right|$. Sufficient uniformity of plate's temperature requires that the amplitude of the temperature variations, $T(z=0)$, at the plate surface do not exceed the maximum acceptable value $\delta_{c}$ (see Sec. II),

$$
H(q a)>a G_{p} / \delta_{c} .
$$

A short physical discussion could be useful at this point. $H(q a)$ greatly simplifies in the limiting cases of thin or thick plates. We are in the thin plate limit if $|q a| \ll 1$ (indeed, $|q a|<0.5$ is sufficient). It corresponds to a quasilinear temperature profile in the plate, due to a sufficiently long range and slow perturbation. Then $H(q a) \simeq\left|q^{2} a^{2}\right|$. We shall see in the next section that probably most helium experiments are in this case.

The thick plate case corresponds to short range or rapid perturbations. As $\operatorname{Real}\left(q^{2} a^{2}\right)>0$, we have $\operatorname{Real}(q a)$ $>|\operatorname{Im}(q a)|$. Thus the thick plate case always corresponds to $\operatorname{Real}(q a)>1$, and $\left|e^{q a}\right| \gg\left|e^{-q a}\right|$. Then $H(q a) \simeq|q a|$. It corresponds to the temperature perturbation being limited to a thin "skin depth" compared to the plate thickness $a$.

These two limiting cases well represent many experiments or practical situations, as shown on Fig. 1.

\section{COMPARISON TO EXPERIMENTS}

A possible order of magnitude of $G_{p}$ is the average gradient $G_{o}$ in the plate,

$$
G_{p} \simeq G_{o} \simeq\left(\lambda_{f} / \lambda_{p}\right) N u(\Delta / h),
$$




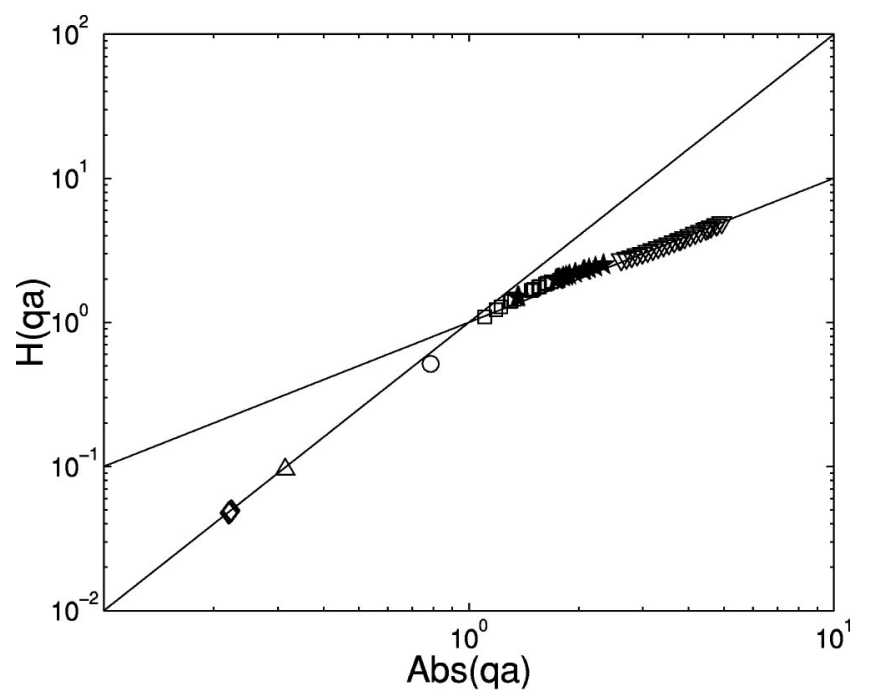

FIG. 1. The value of $H(q a)$, as function of $|q a|$, for various experiments. Symbols are explained in the caption of Fig. 2, after the presentation of these experiments. The two lines correspond, respectively, to $|q a|$ and $|q a|^{2}$.

where $\lambda_{f}$ (respectively, $\lambda_{p}$ ) is the fluid (respectively, plate) thermal conductivity, and $h$ is the height of the RayleighBénard cell.

For $\delta_{c}$ we can choose the typical temperature fluctuation in the center of the cell, which we estimate as

$$
\delta_{c}=\frac{\left\langle u_{z} \theta\right\rangle}{\left\langle\left|u_{z}\right|\right\rangle},
$$

where $u_{z}$ is the vertical velocity of the fluid, and $\theta$ its typical temperature fluctuation. \langle\rangle averages across the center horizontal section of the cell. For large $\mathrm{Nu}$, the average $\left\langle u_{z} \theta\right\rangle$ is proportional to the heat flux, thus to Nu. $\left\langle\left|u_{z}\right|\right\rangle$ is proportional to the Reynolds number. We have

$$
\delta_{c}=\Delta(\mathrm{Nu} / \mathrm{Re} \operatorname{Pr}) .
$$

Using Eq. (9) and Eq. (11) we obtain

$$
\frac{a G_{p}}{\delta_{c}}=\frac{a}{h} \frac{\lambda_{f}}{\lambda_{p}} \operatorname{Re} \operatorname{Pr} .
$$

We have now to estimate the horizontal wave number $k$ and the frequency $\omega$. In real experiments, the plate has a finite diameter $d=\Gamma h$. To simulate it, we take $k \simeq \pi / d$ $=\pi / \Gamma h$ (see the end of the following section for further discussions). For $\omega$, we take the boundary layer typical frequency: $\omega \simeq \kappa_{f}(2 N u / h)^{2}$. Thus, we can estimate $q a$ through

$$
(q a)^{2}=\frac{a^{2}}{h^{2}}\left(\frac{\pi^{2}}{\Gamma^{2}}-i \frac{4 \kappa_{f}}{\kappa_{p}} \mathrm{Nu}^{2}\right) .
$$

Using Eqs. (8) and (12), the criterion for the plates being sufficiently good to allow the ultimate regime to develop, is

$$
\mathrm{Cr}=\frac{H(q a) \delta_{c}}{a G_{p}}=\frac{H(q a)}{\operatorname{Re} \operatorname{Pr}} \frac{h}{a} \frac{\lambda_{p}}{\lambda_{f}}>1 .
$$

Generally, we must consider four cases. As discussed earlier, the plate can be thin $(|q a|<1)$ or thick $(|q a|>1)$, and $(q a)^{2}$ can be dominated by its real or its imaginary part. Following Eq. (13), the imaginary part dominates if

$$
\mathrm{Nu}>\mathrm{Nu}_{c}=\frac{\pi}{2 \Gamma} \sqrt{\frac{\kappa_{p}}{\kappa_{f}}} .
$$

We detail each of these four cases below.

(i) $|q a|<1$ and $\mathrm{Nu}<\mathrm{Nu}_{c}$. Using Eqs. (13) and (14), and the approximation $H(q a) \simeq|q a|^{2}$, we have

$$
\mathrm{Cr}=\frac{\pi^{2}}{\Gamma^{2}} \frac{a}{h} \frac{\lambda_{p}}{\lambda_{f}} \frac{1}{\operatorname{Re} \operatorname{Pr}} .
$$

In these conditions, a good plate must be not too thin, of good thermal conductivity compared to the fluid ones.

(ii) $|q a|>1$ and $\mathrm{Nu}<\mathrm{Nu}_{c}$. This corresponds to a huge thickness of the plate, of the order of its diameter or larger as $q \simeq k$,

$\mathrm{Cr}=\frac{\pi}{\Gamma} \frac{\lambda_{p}}{\lambda_{f}} \frac{1}{\operatorname{Re} \operatorname{Pr}}$.

In these first two cases, the uniform temperature of the plate is due to its great thermal conductivity.

(iii) $|q a|<1$ and $\mathrm{Nu}>\mathrm{Nu}_{c}$. Here

$$
\mathrm{Cr}=4 \frac{a}{h} \frac{C_{p}}{C_{f}} \frac{\mathrm{Nu}^{2}}{\operatorname{Re} \operatorname{Pr}}
$$

where $C_{p}$ (respectively, $C_{f}$ ) is the plate (respectively, fluid) heat capacity per unit volume.

(iv) $|q a|>1$ and $\mathrm{Nu}>\mathrm{Nu}_{c}$. The condition for the plate being thick is here less drastic. It must be thicker than the thermal skin depth. Now

$$
\mathrm{Cr}=\frac{2 \mathrm{Nu}}{\operatorname{Re} \operatorname{Pr}} \sqrt{\frac{\lambda_{p} C_{p}}{\lambda_{f} C_{f}}} .
$$

Here, the important quantity is the product $\lambda_{p} C_{p}$, which can be called the "thermal impedance" of the plate.

For low temperature helium experiments, with copper plates, typical values are $\kappa_{p} \simeq 1 \mathrm{~m}^{2} / \mathrm{s}$, and $\kappa_{f} \simeq 10^{-8} \mathrm{~m}^{2} / \mathrm{s}$, so the real part of $(q a)^{2}$ dominates up to $\mathrm{Nu}$ of order $10^{4}$. On the other hand, for water experiments with copper plates at room temperature, typical values are $\kappa_{p} \simeq 10^{-4} \mathrm{~m}^{2} / \mathrm{s}$ and $\kappa_{f}$ $\simeq 10^{-7} \mathrm{~m}^{2} / \mathrm{s}$. For $\mathrm{Nu}$ values larger than 30 the imaginary part dominates. The same conclusion holds for pressurized gases and mercury, where $\kappa_{f}$ is even larger.

Figure 2 displays the real and imaginary parts of $q a$ for different experiments. Both the Chicago group $(\triangle)$ (Ref. 3) and the Grenoble group (○) (Ref. 9) published tables with the helium properties corresponding to each of their points. The Oregon group $(\diamond)$ did not, but we can use the Grenoble helium properties, together with the Oregon cell's size, to calculate the equivalent $\mathrm{Ra}$. We then calculated the $\mathrm{Nu}$ from their formula, and Re from the following formula, valid for $\operatorname{Pr}>0.6$ (Ref. 9 and references therein):

$$
\operatorname{Re} \operatorname{Pr} \simeq 0.206 \mathrm{Ra}^{0.49} \operatorname{Pr}^{0.3} \quad \text { (High Pr). }
$$

The heat capacity of copper is well known. ${ }^{16}$ The Chicago group used commercial copper whose heat conductivity is generally estimated as $60 T \mathrm{~W} / \mathrm{mK}$, where $T$ is the absolute 


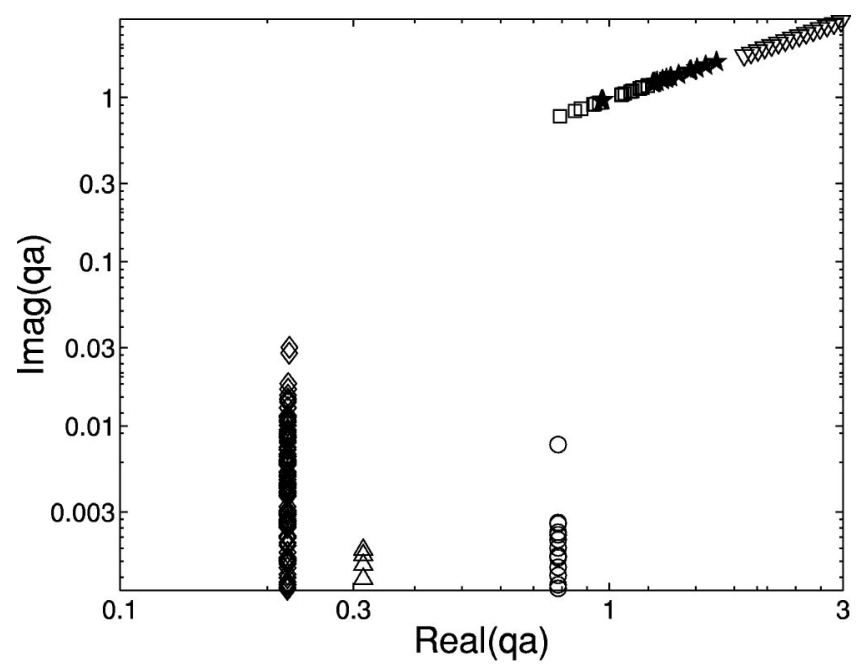

FIG. 2. The parameter $q a$ for different experiments. $\star$, Pressurized gases experiment (Ref. 17). $\bigcirc$, Grenoble points. $\triangle$, Chicago points (Ref. 3). $\square$, Water experiment (Ref. 13). $\nabla$, Mercury experiment (Ref. 18). $\diamond$, Same fluid properties as Ref. 9, but using the cell characteristics of Ref. 5.

temperature, in Kelvin. The Grenoble group used annealed, oxygen-free high-conductivity (OFHC) copper, and measured in situ its thermal conductivity to be $240 T \mathrm{~W} / \mathrm{mK}$. The Oregon group did not measure the thermal conductivity of its plates in situ, but also used anealed OFHC copper. Thus we use the same thermal conductivity.

We also present the results for three room temperature experiments, one with water, one with mercury, and one with pressurized gases $\left(\mathrm{N}_{2}\right.$ and $\left.\mathrm{Ar}\right)$. The water experiment $(\square)^{13}$ has $3 \mathrm{~cm}$ thick copper plates, and an average temperature of $80^{\circ} \mathrm{C}(\mathrm{Pr}=2)$. The pressurized gases experiment $(\star)$ (Ref. 17) has $1.27 \mathrm{~cm}$ thick aluminum plates. The authors published tables with the gas and pressure corresponding to each point. For the mercury experiment $(\nabla)$ (Ref. 18), the difficulty is to know where the transition toward the ultimate regime should take place anyway, as it has never been observed in any low Prandtl number experiment. For simplicity, we assume that it corresponds to the same Reynolds number than for the Grenoble experiment (Ref. 9), that is $R e=6$ $\times 10^{4}$. We estimate the Nusselt and Reynolds number through the correlations proposed by the authors of the experiment, ${ }^{18}$

$$
\mathrm{Nu} \simeq 0.111 \mathrm{Ra}^{0.29}, \quad \mathrm{Re} \simeq 6.24 \mathrm{Ra}^{0.44} \quad \text { (Mercury). }
$$

The Re formula is based on time lag measurements between two temperature probes with vertical separation. Helium experiments ${ }^{3,9}$ [see Eq. (20)] used the same method, which well corresponds to the Reynolds we used to estimate $\delta_{c}$ [Eqs. (10) and (11)]. Sano communicated to the authors of this paper the recent direct velocity measurements at high $\mathrm{Ra}$, with an ultrasonic technics, giving rather small values when compared to this formula (see the web page of the group: http://daisy.phys.s.u-tokyo.ac.jp/paper_e.htm).

Figure 3 shows $\mathrm{Cr}=H(q a) \delta_{c} / a G_{p}$ versus $\mathrm{Ra}$ for the experiments shown in Fig. 2. Niemela et al. ${ }^{6}$ recently remarked that, while the slope $\partial \ln \mathrm{Nu} / \partial \ln \mathrm{Ra}$ of the Grenoble data is large $(\simeq 0.4)$ between $\mathrm{Ra}=10^{11}$ and $\mathrm{Ra}=10^{13}$, it de-

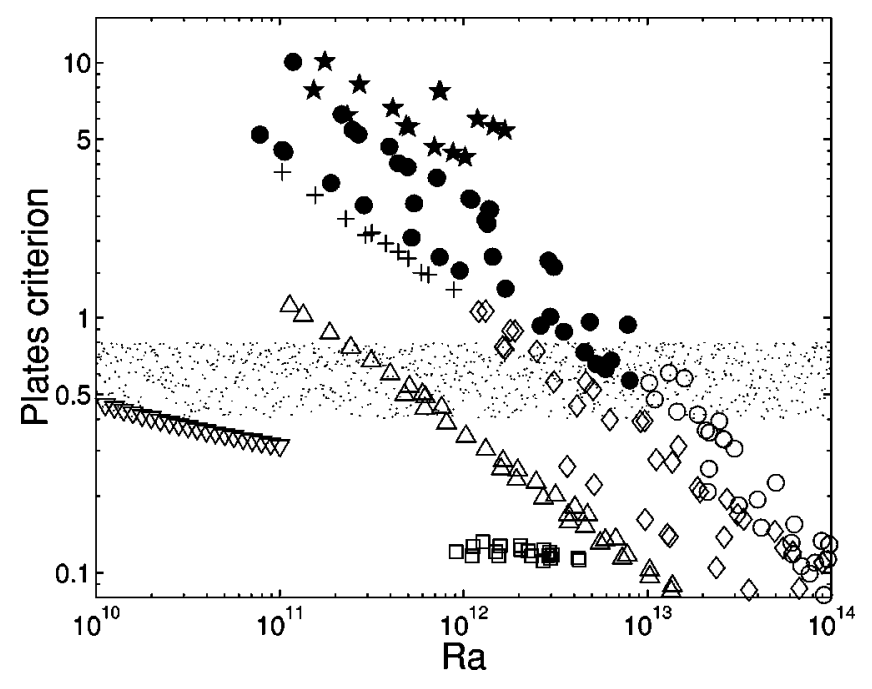

FIG. 3. Value of the plate criterion Cr versus Rayleigh number for various experiments. $\star$, The pressurized gases experiment. $\bullet$, Grenoble points in the ultimate regime (Ref. 9). $\bigcirc$, Grenoble points not in the ultimate regime. $\triangle$, Chicago points (Ref. 3). $\square$, Water experiment (Ref. 13). $\nabla$, Mercury experiment (Ref. 18). + and $\diamond$, the same fluid properties as Ref. 9, but with the cell characteristics of Ref. 5. + corresponds a larger $\mathrm{Nu}$ than in the Grenoble experiment, thus a larger $\mathrm{Nu}$ than the ultimate regime (see text).

creases at higher $\mathrm{Ra}$. It is compatible with $1 / 3$ between $\mathrm{Ra}$ $=10^{13}$ and $\mathrm{Ra}=10^{15}$. We interpret this decrease as $\mathrm{Cr}$ decreasing below its critical value at $\mathrm{Ra}=10^{13}$. We thus distinguish the points below this threshold (solid symbols) which should correspond to the ultimate regime, from the points above (open symbols) which should not.

On the other hand, the $\mathrm{Nu}$ values of the Oregon data ${ }^{5}$ exceed the ultimate regime Grenoble values ${ }^{9,19-21}$ up to Ra $=10^{12}$. Thus, for $\mathrm{Ra}<10^{12}$, a transition to the ultimate regime would make their $\mathrm{Nu}$ values decreasing with $\mathrm{Ra}$ increasing, which is unusual. For this reason, the points below $\mathrm{Ra}=10^{12}$ are distinguished and represented by + .

Figure 3 shows a critical value of $\simeq 0.6$ for $\mathrm{Cr}$, that could explain the discrepancies between the Chicago, Grenoble, and Oregon experiments. Indeed, the Oregon data present a feature close to $R a=10^{12}$, visible in the Kadanoff discussion of the problem. ${ }^{22}$

For the mercury experiment, the direct ultrasonic velocity measurements give $\mathrm{Cr}$ values just above the threshold, which disagrees with other mercury values. It shows the importance of using the same method for determining Re when comparing various experiments. If we except these points, the water and mercury experiments are below the threshold (in accordance with observations ${ }^{13,18}$ ). The mercury one is just below so different cell designs might exceed $\mathrm{Cr}^{23} \mathrm{How}-$ ever, our conclusion partly depends on arbitrary choices. A larger value for $k$ (e.g., $k=2 \pi / d$ instead of $k=\pi / d$ ) would push all the helium experiments ${ }^{3-5}$ to higher values (4 times larger in the example given), and we would have to raise the threshold (e.g., to $\mathrm{Cr}=2.5$ ) further from the mercury values.

For the pressurized gases experiment, ${ }^{17}$ the $\mathrm{Cr}$ values are definitely larger than the threshold. The authors mentioned no transition, but their data are in perfect agreement with the Grenoble ones (see Fig. 4). We thus consider that they would have evidenced the transition if they could have explored a 


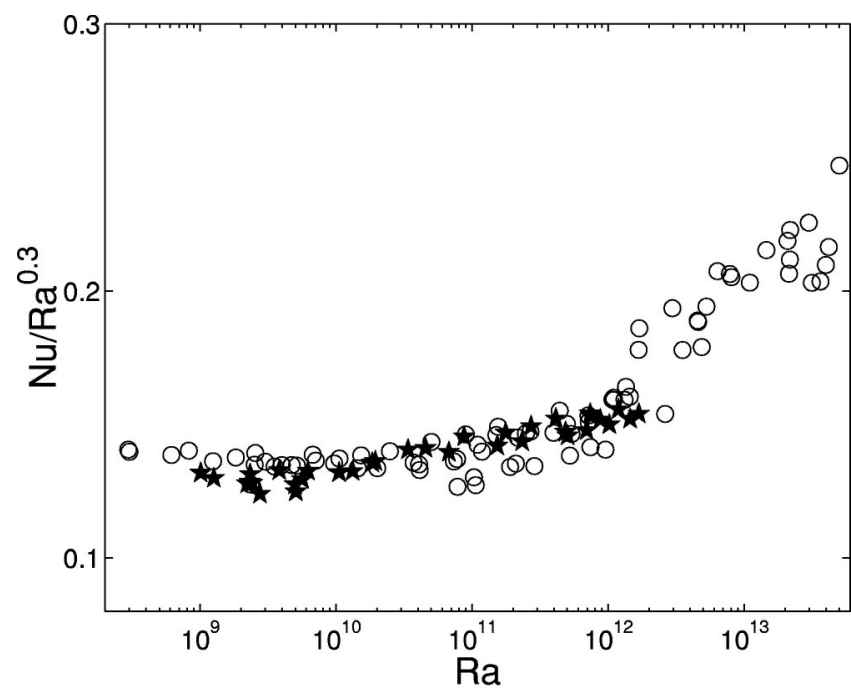

FIG. 4. Comparison between the pressurized gases experiment $(\star)$ (Ref. 17) and the Grenoble one (O) (Ref. 9).

larger Ra range. Indeed, the slope $\partial \ln \mathrm{Nu} / \partial \ln \mathrm{Ra} \simeq 0.327$ they report is larger than the Chicago or Oregon ones.

\section{CONCLUDING REMARKS}

The "elusive" character of ultimate turbulence probably results from plate properties. Both the thermal conductivity and the heat capacity are important. To observe the ultimate regime with a poor heat capacity of the plates needs very high conductivity ratio between the plates and the fluid (in the Grenoble experiment, this ratio goes up to $10^{5}$ ) and thick plates. With a low thermal conductivity, a high heat capacity is not sufficient since only the thermal skin depth helps stabilize the temperature.

These results strongly impact natural convection. In general, soils have a low thermal conductivity, with heat capacity comparable to water. Let us assume a soil has both thermal conductivity and heat capacity of water, and the "thick plate" regime, i.e., $H(q a)=|q a|$. Then, Eq. (19) holds,

$$
\mathrm{Cr}=\frac{2 \mathrm{Nu}}{\operatorname{Re} \operatorname{Pr}} \sqrt{\frac{\lambda_{p} C_{p}}{\lambda_{f} C_{f}}} \text {. }
$$

Soil is rough. In such condition, Roche et al. ${ }^{10}$ have shown that, in the ultimate regime, with $\mathrm{Pr}$ close to $1, \mathrm{Nu}$ $\simeq 6 \times 10^{-4} \mathrm{Ra}^{1 / 2}$. Using this value, and Eq. (20), we obtain

$$
\mathrm{Cr} \simeq 2.8 \text {. }
$$

So, natural convection above soils should be in the ultimate regime. $\mathrm{Cr}$ is sufficiently high that this conclusion is independent of any uncertainties mentioned in the preceding section.

Another important application is the convection in liquid metals, e.g., as primary cooling fluid in a nuclear power plant. ${ }^{24}$ We already discussed experiments which have used mercury, ${ }^{18,23}$ some claiming to have reached the point where the ultimate regime should begin. Other liquid metals in general have higher heat conductivities, which makes $\mathrm{Cr}$ smaller. Thus the Kraichnan regime is unlikely to appear with liquid metals.
These results require further studies for confirmation. Plates properties will have consequences in other practical situations.

\section{ACKNOWLEDGMENTS}

The authors wish to thank R. Verzicco and P.-E. Roche for stimulating discussions, and to M. Sano for communicating results prior to publication. This work was supported by the Région Rhône-Alpes, under Contract No. 301491302.

${ }^{1}$ R. H. Kraichnan, "Turbulent thermal convection at arbitrary Prandtl number," Phys. Fluids 5, 1374 (1962).

${ }^{2}$ E. Siggia, "High Rayleigh number convection," Annu. Rev. Fluid Mech. 26, 137 (1994).

${ }^{3}$ X. Z. Wu and A. Libchaber, "Scaling relations in thermal turbulence: The aspect-ratio dependence," Phys. Rev. A 45, 842 (1992).

${ }^{4}$ X. Chavanne, F. Chillà, B. Castaing, B. Hébral, B. Chabaud, and J. Chaussy, "Observation of the ultimate regime in Rayleigh-Bénard convection," Phys. Rev. Lett. 79, 3648 (1997).

${ }^{5}$ J. J. Niemela, L. Skrbek, K. R. Sreenivasan, and R. J. Donnelly, "Turbulent convection at very high Rayleigh numbers," Nature (London) 404, 837 (2000).

${ }^{6}$ J. J. Niemela and K. R. Sreenivasan, "Confined turbulent convection," J. Fluid Mech. 481, 355 (2003).

${ }^{7}$ S. Grossmann and D. Lohse, "Thermal convection for large Prandtl numbers," Phys. Rev. Lett. 86, 3316 (2001).

${ }^{8} \mathrm{H}$. Tenekes and J. L. Lumley, A First Course in Turbulence (MIT Press, Cambridge, MA, 1972).

${ }^{9}$ X. Chavanne, F. Chillà, B. Chabaud, B. Castaing, and B. Hébral, "Turbulent Rayleigh-Bénard convection in gaseous and liquid He," Phys. Fluids 13, 1300 (2001)

${ }^{10}$ P.-E. Roche, B. Castaing, B. Chabaud, and B. Hébral, "Observation of the $1 / 2$ power law in Rayleigh-Bénard convection," Phys. Rev. E 63, 045303 (2001).

${ }^{11}$ R. J. Goldstein and S. Tokuda, "Heat transfer by thermal convection in high Rayleigh numbers," Int. J. Heat Mass Transfer 23, 738 (1979).

${ }^{12}$ S. Ciliberto, S. Cioni, and C. Laroche, "Large-scale flow properties of turbulent thermal convection," Phys. Rev. E 54, R5901 (1996).

${ }^{13}$ S. Chaumat, B. Castaing, and F. Chillà, "Rayleigh-Bénard cells: influence of the plates' properties," in Advances in Turbulence IX, Proceedings of the Ninth European Turbulence Conference, edited by I. P. Castro, P. E. Hancock, and T. G. Thomas (CIMNE, Barcelona, 2002), pp. 159-162.

${ }^{14}$ J. C. R. Hunt, A. J. Vrieling, F. T. M. Nieuwstadt, and H. J. S. Fernando, "Influence of the thermal diffusivity of the lower boundary on eddy motion in convection," J. Fluid Mech. 491, 183 (2003).

${ }^{15} \mathrm{R}$. Verzicco, "Effects of nonperfect thermal sources in turbulent thermal convection," Phys. Fluids 16, 1965 (2004).

${ }^{16}$ R. R. Conte, "Eléments de cryogénie," Masson et Cie Editeurs, 1970.

${ }^{17}$ A. S. Fleischer and R. J. Goldstein, "High-Rayleigh-number convection of pressurized gases in a horizontal enclosure," J. Fluid Mech. 469, 1 (2002).

${ }^{18}$ J. A. Glazier, T. Segawa, A. Naert, and M. Sano, "Evidence against "ultrahard' thermal turbulence at very high Rayleigh numbers," Nature (London) 398, 307 (1999).

${ }^{19}$ This difference should be related to the bimodality discovered by Roche et al. (Ref. 20), and explained by Verzicco et al. (Ref. 21).

${ }^{20}$ P.-E. Roche, B. Castaing, B. Chabaud, and B. Hébral, "Prandtl and Rayleigh numbers dependences in Rayleigh-Bénard convection," Europhys. Lett. 58, 693 (2002).

${ }^{21}$ R. Verzicco and R. Camussi, "Numerical experiments on strongly turbulent thermal convection in a slender cylindrical cell," J. Fluid Mech. 477, 19 (2003).

${ }^{22}$ L. P. Kadanoff, "Turbulent heat flow: Structures and scaling," Phys. Today 54 (8), 34 (2001).

${ }^{23}$ S. Cioni, S. Ciliberto, and J. Sommeria, "Strongly turbulent RayleighBenard convection in mercury: Comparison with results at moderate Prandtl number," J. Fluid Mech. 335, 111 (1997).

${ }^{24}$ The experimental nuclear power plant Superphénix used liquid sodium as cooling fluid. 